\title{
Trauma y Emergencias En El Sur Del Ecuador; Historia, Realidad y Perspectivas
}

\section{Michel B Aboutanos}

Keywords: Sistema de atención de trauma, Nivel bajo de recursos, Ecuador.

How to cite this article: Michel B Aboutanos. Trauma y Emergencias En El Sur Del Ecuador; Historia, Realidad y Perspectivas. Panam J Trauma Crit Care Emerg Surg 2014; 3(3):141-142.

\section{Source of support: Nil}

\section{Conflict of interest: None}

\section{INTRODUCTION}

El Dr Salamea y sus colegas describieron el progreso de la atención del trauma en la región sureste de Ecuador (Ref. Juan C Salamea, Hernan Sacoto, Edgar B Rodas : Trauma Y Emergencias En El Sur Del Ecuador; Historia, Realidad Y Perspectivas. PAJTCCES 3:70-73, 2014).

Se observaron esfuerzos importantes por varios grupos en distintas etapas. Durante todo el proceso, se delinearon algunos aspectos importantes, notablemente; la necesidad de apoyo público y político para el desarrollo de un sistema de atención de trauma regionalizado. Esto es cierto en cualquier lugar, independientemente del estado económico. ${ }^{1}$ En el sureste de Ecuador, ninguno de los primeros esfuerzos en el desarrollo de una iniciativa educativa en toda la región, el entrenamiento básico de atención de trauma, sobre todo y significativamente un registro de trauma regionalizado podría haber sido posible sin el apoyo del Ministerio Provincial de Salud. Para obtener este apoyo, una evaluación del sitio fue necesaria. Inicialmente, en Ecuador este se produjo de manera informal a través de la colaboración entre la Universidad Commonwealth de Virginia y el Ministerio

\footnotetext{
Profesor

del Departamento de Cirugía; Jefe, Cuidado Agudo Servicios quirúrgicos; Director Internacional de Trauma Sistemas de VCU; Programa de Desarrollo, la Virginia Commonwealth University, Virginia, EE.UU

Corresponding Author: Michel B Aboutanos, del Departamento de Cirugía; Jefe, Cuidado Agudo Servicios quirúrgicos; Director Internacional de Trauma Sistemas de VCU; Programa de Desarrollo, la Virginia Commonwealth University, Virginia, EE.UU, e-mail: maboutanos@mcvh-vcu. edu
}

de Salud, y posteriormente a través de una encuesta formal, utilizando las Guías Esenciales de la Atención del Trauma de la OMS, estos esfuerzos de colaboración incluyeron a la Sociedad Ecuatoriana de Trauma y la Sociedad Panamericana de Trauma. El uso del modelo de salud pública para el desarrollo de sistemas de trauma puede ser igualmente útil en países de ingresos altos, así como bajos y medianos. ${ }^{2}$

La clave para el desarrollo temprano de la atención del trauma en la región es la participación temprana de los médicos líderes locales, activistas comunitarios y administradores que fueron ingeniosos y pacientes atreves de todo el proceso, especialmente en la demostración de la necesidad de un cambio en la prestación de atención del trauma. La colaboración a largo plazo entre el ministerio provincial de salud de Morona Santiago, las universidades locales de Cuenca y Azuay, la Fundación CINTERANDES y el Programa Internacional de Desarrollo de Sistemas de Trauma de la Universidad Virginia Commonwealth (ITSDP) fueron vitales. Facilito el establecimiento de la infraestructura para una sistema regional de trauma en la región sureste de Ecuador, incluyendo los programas de prevención de lesiones, el desarrollo del Curso Básico de Trauma para la educación practica a nivel pre-hospitalaria y hospitalaria, y lo más importante el soporte político para el cambio a nivel del ministerio de salud. ${ }^{3}$

La colaboración de una universidad académica de Estados Unidos importante en este proceso tampoco debe subestimarse. Presta legitimidad, experiencia académica significativa, logística y apoyo financiero, y lo más importante relaciones a largo plazo que son igualmente beneficiosas. De la misma manera, muchas de las inversiones de la Universidad fueron igualmente gratificantes e importantes. Las lecciones aprendidas sobre todo en el desarrollo de un registro de trauma para los lugares de ingresos bajos y medianos fueron instrumentales en el desarrollo a través de los esfuerzos de la universidad, de un registro de trauma modular que fue adoptado por la Sociedad Panamericana de Trauma como una herramienta útil recopilación de datos e implementadas en la región de América Latina. ${ }^{4}$

Al mismo modo, como se menciona en el artículo, un Curso Básico de Trauma, diseñado para ofrecer educación básica y entrenamiento para el manejo inicial del paciente 
herido en sitios de bajos recursos, ha sido adoptado como el curso Básico de Trauma oficial de la SPT y ha sido administrado en toda la región, incluyendo Paraguay, Colombia, Chile y Panamá.,

Tal vez el aspecto más importante no mencionado por los autores, son en si los mismos autores. La migración calificada y la fuga de ideas ha sido una gran preocupación para los países de ingresos medios y bajos, incluyendo a Ecuador. Cuando las personas que estudiaron y completaron su educación en el extranjero no regresan a su país de origen, esto se traduce en una pérdida significativa y de gran valor para el país de origen. Todos los autores de este articulo excelente tuvieron la oportunidad de adquirir una formación invaluable y habilidades fuera del Ecuador, con gran oportunidad de permanecer en el extranjero. Sin embargo su compromiso de regresar a Ecuador, y abrazar el desarrollo de la atención del trauma en su región a pesar de todos los desafíos y vejación, es la verdadera medida del éxito, y será la mejor recompensa de sus esfuerzo. Su trabajo describe su aprecio por los esfuerzos realizados en el desarrollo de la atención del trauma en su región, pero lo más importante habla de un plan estratégico para construir el futuro de la atención del trauma en Ecuador, comenzando con la Liga Académica de Trauma Estudiantil, al mismo tiempo preparando redes sostenibles para el desarrollo de infraestructura: un esquema para otras regiones y locales.

\section{REFERENCIAS}

1. Bazzoli GJ. Community-based trauma system development: key barriers and facilitating factors. J Trauma 1999 Sep Suppl;47(3):S22-S24.

2. Model Trauma System Planning and Evaluation. The Health Resources and Services Administration. US Department of Health and Human Services 2006.

3. Aboutanos M, Mora F, Duong M, et al. Telemedicine applications and rural trauma system development in Latin America: Ecuador's experience. Journal of eHealth Technology and Application 2007;(5)2. Available at: www. tsj.gr.jp; Accessed on 1/12/2005.

4. Ordóñez C, Pino L, Tejada J. Experience of two first level hospitals in the southwest region of Colombia on the implementation of the Panamerican Trauma Society International Trauma Registry. Rev Col Bras Cir 2012;39(4): 255-262.

5. Aboutanos M, Rodas E, Aboutanos S, et al. Trauma education and care in the jungle of Ecuador, where there is no advanced trauma life support. J Trauma 2007 Mar;62(3):714-719.

6. Panamerican Trauma Society. Basic Trauma Course. Available at: www.panamtrauma.org; accessed on 1/12/15. 